\title{
Low-dose imaging of metal-organic frameworks and study of their electron-beam stability
}

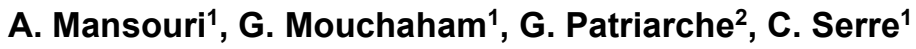 \\ ${ }^{1}$ Institut des Matériaux Poreux de Paris, Ecole Normale Supérieure, ESPCI Paris, CNRS, PSL University, 75005 Paris, France \\ ${ }^{2}$ Center for Nanoscience and Nanotechnology, C2N UMR 9001, CNRS, Université Paris Sud, Université Paris Saclay, Palaiseau, \\ France
}

asma.mansouri@ens.psl.eu

Metal-organic frameworks (MOFs) are hybrid (organic/inorganic) crystalline porous solids intensively studied for their potential applications in different domains related to energy, environment or health [1]. Diffraction-based techniques are one of the major methods for the crystal structure determination of MOFs, providing a better understanding of their unique properties. However, the in formation obtained is the averaged periodic structure, while in some cases - such as adsorption and catalysis - the local structural features (ie., crystal surfaces, interfaces, the presence of guest molecules or defects) are key elements, which can be visualized using high-resolution TEM methods. However, MOFs are among the most beam-sensitive materials and can be easily damaged after exposure to a few electrons $/ \AA^{2}$. Therefore, only few successful studies of HRTEM imaging were reported, so far, focusing on just a handful of MOFs [2]. These recent studies have demonstrated that using a direct detection electron counting camera (DDEC) allows performing HRTEM imaging of MOFs with extremely low dose rates (as low as $4 \mathrm{e}^{-/} / \AA^{2} . \mathrm{s}$ ). Thus, individual inorganic clusters could be visualised, while in some cases, the organic linkers could be also resolved [3].

The prediction of the electron-beam stability of a MOF nanocrystal is a difficult task since many structural, morphological or instrumental parameters might be taken into account and could have interconnected effects (Figure 1a). This work aims at identifying the most prominent parameters and assessing their influence on the stability of these objects when exposed to the beam and under given operating conditions. Several known MOFs were imaged by low-dose HRTEM (Figure 1b) enabling the investigation of the effect of the voltage, the particle size and orientation, the presence of guest molecules, as well as the nature and the geometry of the organic and the inorganic moieties. The threshold cumulative electron dose that a MOF can withstand before being completely damaged is determined by monitoring the fading of the spots on the fast Fourier transform images calculated after different exposure times. It has been found that the size of the particle does not have as much impact on the stability as its orientation and its degree of crystallinity, while the presence of guest species encapsulated in the pores has been found to significantly improve the stability. The comparison of the lowest and highest threshold electron doses measured for particles oriented along random off-zone axes of a series of aluminiumbased and titanium-based MOFs (Al-MOFs and Ti-MOFs) is given in Figure 1b. The obtained results and the influence of the different features and parameters (ie., linker connectivity, nuclearity of the inorganic building unit, the oxidation state of the metal ions, etc.) on the electron beam stability of these MOFs will be presented. Besides, low-dose HRTEM imaging realised on new in-house MOFs and its use for structure resolution will be also highlighted.

a)

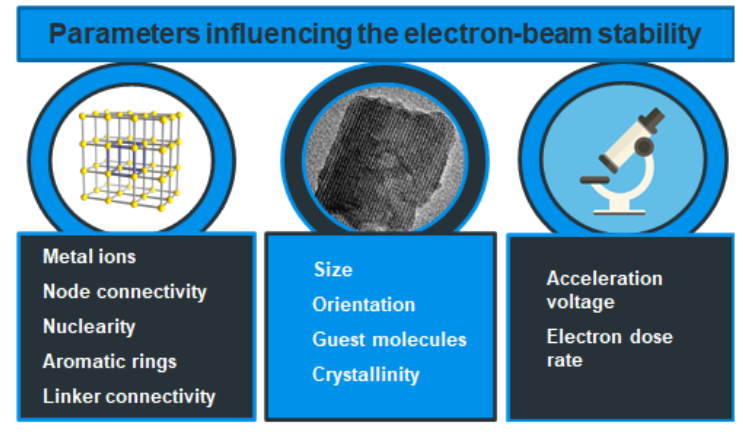

b)
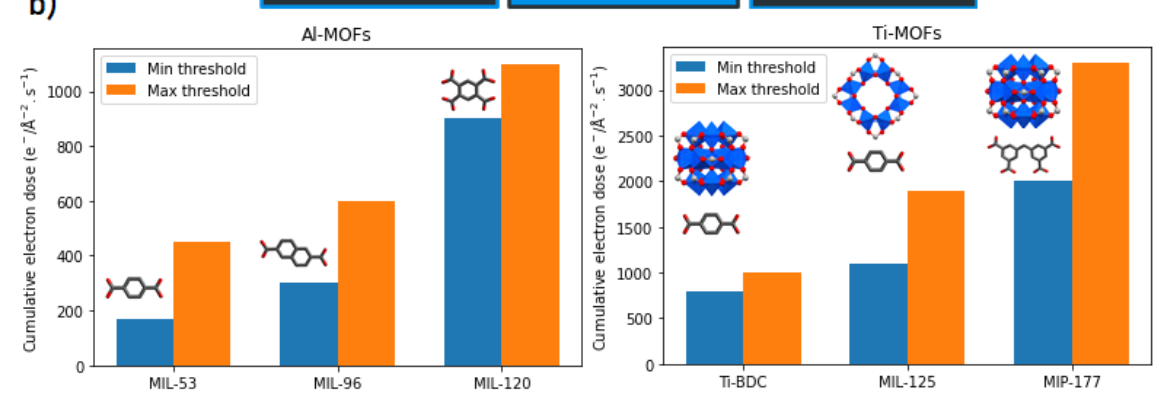

Figure 1. a) Schematic of the parameters that could influence the electron beam stability of a MOF. b) Summary of the lowest and the highest threshold electron doses $\left(\mathrm{e}^{-} / \AA^{2} . \mathrm{s}\right)$ measured on off-zone axis particles of a series of Al-based and Ti-based MOFs. 


\section{PS-28-1}

Poster Session

[1] Wang, S., \& Serre, C. (2019). ACS Sustainable Chemistry \& Engineering, 7(14), 11911-11927..

[2] Liu, L., Zhang, D., Zhu, Y., \& Han, Y. (2020). Commun. Chem. 3(1), 1-14.

[3] Zhu, Y., J. Ciston, L., Zheng, B., Miao, X., Czarnik, C., Pan, Y., Sougrat, R., \& Han, Y, Nat. Mater. (2017), 16, $532-536$.

Keywords: Metal-organic frameworks; Low-dose high-resolution TEM; Beam sensitive materials; Structure determination

The authors acknowledge the Region Ile-de-France in the framework of DIM ResPore for the financial support. 